\title{
La Recherche
}

\section{Lusomondialisation?}

L'économie politique du Brésil de Lula

\section{Lusomundialização?}

A economia política do Brasil de Lula

\section{Lusoglobalization?}

The political economy of Lula's Brazil

sous la direction de / sob a direcção de / edited by

B. Lachartre et P. Salama 


\section{INTRODUCTION}

$\mathrm{D}$ ouze ans après la publication d'un dossier sur les transitions libérales en Afrique lusophone ${ }^{1}$, la revue Lusotopie se propose de réfléchir sur l'insertion des pays lusophones dans l'économie-monde, désignée ici sous le terme de «lusomondialisation ». Au-delà des mécanismes et ressorts aujourd'hui bien identifiés, propres à cette nouvelle phase du développement du capitalisme et à son renouvellement, il s'agit de cerner l'impact et les spécificités de l'économie globalisée sur certains pays de l'aire lusophone qui intéressent la revue.

Le cas du Brésil a été choisi et approfondi pour la richesse qu'il offre à l'analyse de cet espace où se croisent et se heurtent, le plus souvent, économie et politique. Quelle réalité la mondialisation recouvre-t-elle dans un pays tel que le Brésil $^{2}$ ? Puisqu'il est clair qu'aucun pays ne se tient désormais à l'écart de l'économie mondialisée, la question posée est celle d'évaluer le degré d'insertion recherché ou atteint par chacun d'eux, et d'en mesurer les effets sur les systèmes économiques et politiques qui leur sont propres. Plus généralement, la libéralisation des marchés a-t-elle été pour ces pays un succès en termes de croissance, de modernisation, de vulnérabilité, de meilleure cohésion sociale ? Suite à la « décennie perdue $»^{3}$ des années 1980, qui a vu la mise en application des accords avec le Fonds monétaire international (FMI), avec les résultats désastreux du point de vue économique et social que l'on sait, comment fonctionne aujourd'hui le modèle économique néolibéral dans un contexte aussi contrasté sur le plan politique et économique que le Brésil ? En quoi son application et ses résultats contribuent-ils à alimenter la controverse au sujet du gouvernement de Lula?

Les préconisations du consensus de Washington (1990), qui ont, depuis, rénové la doctrine et redirigé les économies afin d'éradiquer l'inflation et d'assurer le service de la dette extérieure, ont été appliquées au Brésil avec un zèle remarquable, surprenant pour un pays gouverné par un leader politique élu sur une base populaire. Quels sont les effets, aujourd'hui, de la discipline fiscale, mais aussi de la réorientation des dépenses publiques en faveur des infrastructures, de la santé, de l'éducation, de la lutte contre la pauvreté ?

${ }^{1}$ M. CAhen (ed.), «Transitions libérales en Afrique lusophone » : 85-358, Lusotopie (Karthala, Paris), 1995.

${ }^{2}$ Lusotopie a abondamment traité d'autres aspects de la réalité brésilienne dans ses différentes livraisons. Voir en particulier les dossiers «L'oppression paternaliste au Brésil» (1996), «Des protestantismes en "lusophonie catholique" " (1998), "Dynamiques religieuses en lusophonie contemporaine » (1999), "Violences et contrôle de la violence au Brésil, en Afrique et à Goa » (2003), «Médias, pouvoirs et identités " (2004) [textes intégraux disponibles sur le site $<$ www.lusotopie.sciencespobordeaux.fr $>$ ]. Voir aussi l'article sur les élections récentes au Brésil, de S. Monclaire, «Lula II, un vote de reconnaissance », XIV (1), juin 2007 : 3-68.

${ }^{3}$ Expression empruntée à la Cepal (Commission économique pour l'Amérique latine et les Caraïbes) pour qualifier les années 1980 au cours desquelles une très grande majorité des économies latino-américaines ont connu stagnation, inflation et extension de la pauvreté. 
Au-delà du Brésil, que nous enseigne l'étude de ce cas particulier pour la compréhension du phénomène de mondialisation? En quoi les réflexions qu'en retirent les auteurs peuvent-elles amener à une meilleure compréhension du phénomène à l'échelle d'autres pays, d'autres continents? Tel est le propos de ce dossier, qui, on peut l'espérer, sera suivi par des travaux du même type, portant sur d'autres aires lusophones. Mais le fait d'avoir dû traiter le Brésil à part témoigne en luimême de la difficulté à appréhender, du point de vue de l'économie politique, la lusophonie (et la lusotopie) ${ }^{4}$ au temps de la mondialisation, la «lusomondialisation ».

\section{La lusophonie dans la mondialisation?}

L'origine de ce dossier réside dans une interrogation majeure sur les modes particuliers d'implantation et de développement de l'économie globalisée dans des aires ayant historiquement connu le même acteur colonial, mais connaissant aujourd'hui des contextes nationaux et géopolitiques si différents, avec leurs histoires économiques et sociales propres, l'adoption de doctrines économiques particulières dans la seconde moitié $\mathrm{du} \mathrm{xx}^{\mathrm{e}}$ siècle (développement autocentré, substitution des importations, économie planifiée, etc.) et qui sont donc entrés à l'orée du $\mathrm{xxI}^{\mathrm{e}}$ siècle dans l'ère de la nouvelle économie avec des héritages diversifiés. Ce sont ces ancrages particuliers, ces articulations du système globalisé avec les économies nationales qu'il s'agit de dégager dans un paysage mondialisé qui n'est uniforme qu'en apparence, où, plus que les similitudes maintes fois répertoriées, il s'agit de distinguer en quoi des historicités distinctes provoquent en réalité des effets sensiblement divergents. Le comparatisme «intra-lusophone » n'a d'ailleurs de sens que complété par l'approche comparative avec d'autres pays émergents, du même continent (Mexique, Argentine) ${ }^{5}$ ou du continent asiatique (Chine, Corée du Sud... $)^{6}$. Si la mondialisation est une tendance plus ou moins généralisée du capitalisme contemporain, l'apposition de préceptes uniformes (ouverture des marchés, importance croissante du secteur financier, libéralisation des taux d'intérêts, privatisation des entreprises publiques, marchandisation des services publics...) sur des réalités diverses, voire divergentes, ne provoque pas nécessairement un rapprochement des situations et, à l'inverse, peut accentuer les disparités de trajectoires. L'uniformité imposée à la diversité produit de l'inégalité. Il est donc nécessaire d'y regarder de près. Qu'est-ce qui différencie ces économies et pourquoi réagissent-elle différemment à la mondialisation? Qu'est-ce qui explique qu'elles atteignent des taux de croissance si différents alors qu'elles appliquent grosso modo les mêmes recettes? À quoi tient la volatilité de cette croissance insaisissable, et quels sont ses effets sur la pauvreté ? En quoi la structuration sociale (existence et

\footnotetext{
${ }^{4}$ Rappelons que le concept de «lusotopie », forgée par notre revue, définit les aires ou communautés issues, à divers degrés, de l'histoire et de la colonisation portugaises, quelle(s) que soi(en)t leur(s) langue(s) officielle(s) ou nationale(s) aujourd'hui.

${ }^{5}$ P. Salama, « Argentine, Brésil, Mexique : libéralisation et nouvelles vulnérabilités », L'Économie politique (Alternative économique), 2006, IV (32): 55-68.

${ }^{6}$ P. Salama, Le défi des inégalités, une comparaison économique Amérique latine/Asie, Paris, La Découverte, 2006.
} 
comportements de classes sociales bien distincts selon les pays) s'avère-t-elle décisive dans les performances du modèle appliqué?

Qu'en disent les chercheurs ici réunis? Ils indiquent - moult comparaisons et diagrammes à l'appui - qu'il est nécessaire de prendre en compte certains facteurs déterminants non directement économiques tels le niveau des inégalités sociales (mesurées sous divers angles) ou le niveau éducatif, et la complexité de leur interrelation, pour comprendre et mesurer les effets de la globalisation dans une société ainsi que les difficultés qu'elle rencontre à les surmonter à plus ou moins long terme.

Par ailleurs, face aux « défis des inégalités » ${ }^{7}$, à la pauvreté et, en ce qui concerne le Brésil, aux promesses électorales faites pour les combattre et aux rapports sociaux qui structurent cette société, il s'agit également de s'interroger sur les marges de manœuvre dont disposent les politiques, sur leurs capacités à gérer le coût social des mesures de convergence, les contradictions qu'elles entraînent, les oppositions d'intérêt qu'elles soulèvent. Quels sont donc la place et le rôle de l'État (politiques industrielles, programmes de lutte contre la pauvreté, réforme agraire...)? De quels leviers dispose-t-il (entreprises publiques, secteur des infrastructures et des communications, commerce extérieur...) pour tourner à leur avantage les potentialités existantes et fixer la croissance à un taux suffisant pour surmonter les crises?

«La globalisation : responsable ou bouc émissaire? » est la question qui traverse les articles regroupés dans ce dossier sur l'économie politique du Brésil de Lula. Les auteurs (des économistes, un sociologue, un politiste et un agronome) développent ici pour la plupart une vision critique du gouvernement de Lula, quel que soit l'angle d'attaque de leurs analyses (la pauvreté et sa «métamorphose », la politique agricole et le développement rural, la politique sociale...). Sur tel ou tel point, ils indiquent que des alternatives aux politiques suivies existent... même si elles ne sont pas retenues.

Il en résulte dans l'ensemble une mise en cause, un doute sur la volonté politique réelle de Lula et de son équipe de tenir les promesses faites ainsi qu'une démonstration alarmante des risques de non-redressement économique et de persistance des inégalités.

Cela mène à questionner la nature du gouvernement Lula, la base sociale sur laquelle il s'appuie (article de A. Boito Jr), les chances qu'il a de conserver la confiance de son électorat populaire. Malgré tout, les relativement faibles performances économiques et sociales de sa première mandature n'ont guère eu d'effets quantitatifs sur les résultats électoraux de sa candidature à un second mandat, même si leur analyse détaillée montre des inflexions sociales notables ${ }^{8}$.

Les analyses et prises de position critiques et contrastées présentées dans ce dossier témoignent en tout cas de la vigueur des débats qui se poursuivent au Brésil sur l'ensemble des questions évoquées et du maintien de la tradition d'une économie politique critique dans ce pays.

\footnotetext{
7 Ibid.

8 S. Monclaire, op. cit.
} 


\section{Croissance médiocre, vulnérabilité sociale : vers la fin de l'abertura brésilienne?}

Le Brésil a connu ces vingt-cinq dernières années une croissance médiocre, particulièrement faible si on la compare à celle de la Chine, voire aujourd'hui de l'Inde ou de la Russie. Une croissance réduite a pour conséquence une mobilité sociale faible et donc le maintien d'une vulnérabilité sociale forte: la probabilité de passer en effet d'une catégorie sociale à une autre est réduite avec un taux de croissance par habitant faible et donc celle de quitter le monde de la pauvreté lorsqu'on naît pauvre est quasi inexistante, sauf à réduire sensiblement les inégalités de revenu, ce qui n'a pas été le cas. C'est ce qui explique le maintien de la pauvreté à un niveau élevé. À l'inverse, la vulnérabilité économique du Brésil diminue avec la croissance élevée des exportations ${ }^{9}$, l'apparition et l'essor d'un solde commercial excédentaire, suffisant pour financer les intérêts de la dette externe, le paiement des dividendes versés aux actionnaires étrangers et les divers services tels que les assurances, les royalties, les transports. Le poids de la dette par rapport au Produit intérieur brut (PIB), et de son service par rapport aux exportations, diminue et le Brésil progressivement reconquiert une crédibilité auprès des marchés financiers internationaux.

Vulnérabilité sociale maintenue à un niveau élevé et vulnérabilité économique en voie de réduction s'opposent. Certains ont pu penser que la diminution de la vulnérabilité économique allait permettre ensuite de diminuer la vulnérabilité sociale et qu'en quelque sorte ce chemin constituait la voie obligée pour assurer demain une reprise assainie de la croissance ouvrant la voie à une mobilité sociale plus conséquente et donc à un espoir d'amélioration de la situation des plus démunis, nombreux au Brésil. Cette vision gradualiste voire «étapiste» s'est traduite par des échecs. Certes la vulnérabilité économique a diminué mais la cuirasse renforcée reste bien mince face aux tsunamis financiers potentiels, et ce serait une illusion que de penser que le retour relatif de la confiance auprès de marchés financiers internationaux et les réserves accumulées constituent des obstacles suffisants pour faire face à la spéculation financière, d'autant plus qu'en moyenne la globalisation financière a cru plus vite au Brésil que dans la plupart des autres pays.

La financiarisation des activités renforcée par la politique de taux d'intérêt extrêmement élevés freine la croissance des investissements productifs, maintenant le taux d'investissement à un niveau faible, très en deçà de ce qui serait nécessaire pour atteindre une croissance plus importante (article de P. Kliass \& P. Salama). Cette politique s'accompagne d'un taux de change inutilement apprécié - sauf à satisfaire les intérêts des entreprises multinationales et des investisseurs étrangers de manière générale - qui continue à se valoriser (article de A. Boito Jr). L'illusion d'une relation positive entre taux de change fort et assainissement d'une économie, voire reprise d'une croissance est démentie par les faits. Cette politique de taux de change favorise une politique de démantèlement de l'industrie nationale en faveur des importations plus compétitives que la production nationale au lieu de

${ }^{9}$ Selon la Banque mondiale, le degré d'ouverture du Brésil était de $11,7 \%$ en 1990 et a atteint 26,9 \% en 2004, ce qui signifie que la croissance des exportations (et celle des importations) a été beaucoup plus forte que celle du PIB. 
la protéger par un change déprécié dans ses segments les plus potentiellement dynamiques, le temps qu'elle puisse faire face à la compétition internationale. Elle tend à favoriser enfin la production de biens à faible valeur ajoutée et à faible contenu technologique et à rendre plus difficile celle des biens à haute technologie et à forte valeur ajoutée, au point que certains économistes craignent qu'avec cette politique de change le Brésil s'engage peu à peu dans une voie dite de «mexicanisation» de son économie. Cette politique est à l'exact inverse de celle pratiquée par la Chine et l'Argentine aujourd'hui qui, comme on le sait, connaissent des taux de croissance qui n’ont rien à voir avec ceux du Brésil...

Sans aller jusqu'à évoquer la thèse de la désindustrialisation du Brésil, qui se discute dans le pays, on peut considérer qu'il est aujourd'hui à la croisée des chemins. À la différence de l'Argentine, son appareil de production, modernisé, moins intégré que par le passé grâce à une ouverture croissante et à l'importation plus massive de biens d'équipement, reste le socle de l'essor de ses exportations, même si une partie importante s'explique par celle de matières premières en général plus transformées qu'en Argentine. Pour autant la politique économique suivie a freiné la croissance. Le paradoxe est alors double.

Premièrement, l'appareil de production est un des plus modernes d'Amérique latine, les politiques d'austérité dès les années 1980 ont détruit des pans entiers de cet appareil, au prix d'un coût social considérable, mais ont aussi participé à la modernisation de certains de ses segments, ce qui se traduit aujourd'hui par le retour d'un excédent important de la balance commerciale, y compris lorsqu'on soustrait les effets positifs du renchérissement des matières premières. Pour autant, malgré ces excédents et la croissance forte des exportations, le poids du Brésil dans le commerce mondial ne s'est pas accru et tourne autour du $1 \%$ alors que celui de la Chine, en croissance très rapide de ses exportations, est aujourd'hui sept fois plus élevé. Plus grave, la croissance des exportations de produits de haute et de «moyenne-haute» technologies, celles qui sont révélatrices du dynamisme d'une économie, celles qui ont l'élasticité de la demande - par rapport au revenu la plus élevée, ne s'est pas accrue de manière significative et leurs poids dans les exportations manufacturières ont baissé depuis la fin des années 1990 alors qu'on observe une évolution inverse dans les économies asiatiques. À l'inverse, la part des produits de «moyenne-basse » technologie s'est accrue comme le montrent des études récentes de l'Instituto de estudos para o desenvolvimento industrial en mai 2007 et de l'Instituto brasileiro de geografia e estatística.

Deuxièmement, les politiques de change et de taux d'intérêt ont été un succès au plan de la maîtrise de l'inflation, mais elles ont miné la croissance. La politique budgétaire très restrictive en matière d'investissements publics a pris également sa part de responsabilité dans l'institutionnalisation d'une croissance molle sur le long terme, plus ou mois volatile sur le court terme ${ }^{10}$. La poursuite de ces politiques de change, monétaire, budgétaire, condition apparemment d'un retour de la confiance des investisseurs internationaux, freinant la croissance, est à terme de

${ }^{10}$ L'insuffisance des investissements publics réalisés ces dix dernières années dans le domaine de l'énergie bloque au-delà d'un an ou deux la possibilité de maintenir un taux de croissance supérieur à 3 ou $4 \%$, sauf à procéder à des investissements massifs dans ce domaine. 
nature à fragiliser cette confiance pour cette raison. Comme il est impossible de réduire significativement le niveau de pauvreté sans reprise conséquente de la croissance, seules des politiques redistributives pourraient atteindre cet objectif. Or celles qui ont été menées jusqu'à présent sont insuffisantes même si politiquement elles ont pu être valorisées lors des dernières élections présidentielles (article de G. Druck \& L. Filgueiras) ; on considère en effet qu'il y a un rapport de 1 à 15, voire 18 entre les sommes consacrées à la bolsa família (bourse famille) qui concerne à peu près un tiers de la population, et celles versées au titre du service de la dette interne, qui, elles, concernent directement et indirectement de 3 à $5 \%$ de la population. L'allégement de la pauvreté - «concret» pour les pauvres - s'est fait en même temps que se réalisait un enrichissement «abstrait » pour une minorité. L'amélioration, en termes de pouvoir d'achat, aux deux bouts de la distribution des revenus s'est faite au détriment des couches moyennes, d'une inégalité croissante entre ces deux extrêmes (le revenu des uns, les plus nombreux, s'améliorant beaucoup moins que celui des autres, les moins nombreux), au prix d'un ralentissement de la croissance et d'une insuffisance relative de l'ensemble des dépenses sociales fédérales (article de M. Pochmann).

La politique du commerce extérieur du Brésil apparaît souvent comme moins orthodoxe (article de J. Ramos Silva) que celle appliquée au niveau intérieur parce que sur de nombreux points elle participe à une remise en question des anciennes hégémonies qui modèle l'économie-monde. Le Brésil participe dans les négociations internationales à des regroupements avec d'autres économies émergentes comme la Chine, l'Inde, l'Afrique du Sud et trace ainsi de nouvelles configurations, mais il est exact aussi que ces desseins, lorsqu'ils se traduisent par des politiques commerciales «bien comprises » au niveau agricole, mettent en œuvre de nouvelles relations de forces, de nouveaux compromis qui ne sont pas sans conséquences sur l'équilibre des pouvoirs à l'intérieur du pays, notamment dans les relations entre la petite agriculture, négligée et l'«agronégoce » (agro-business) qui reste privilégié (article de É. Sabourin).

\section{À la croisée des chemins}

Le Brésil est donc à la croisée des chemins. Soit il poursuit la même politique et les obstacles s'accumuleront. Les rapports entre les classes et groupes sociaux (analysés par A. Boito Jr), seront davantage fragiles qu'ils ne le sont aujourd'hui et ce qui hier pouvait apparaître comme un gain pour certains légitimant leur appui à la politique du gouvernement, se transformera demain en source d'affaiblissement, de perte et de déficit de légitimation; soit il change de politique quitte à heurter des intérêts. Des signes apparaissent d'une transition vers plus de volontarisme en économie (article de P. Kliass), que ce soit avec le maintien du ministre des finances au gouvernement, le choix du président de la banque de développement économique et social, celui du représentant du Brésil auprès du Fonds monétaire international, et des voix se font davantage entendre en faveur d'une politique économique et sociale orientée vers un nouveau «développementalisme ».

Ce numéro de la revue Lusotopie s'est donné pour objectif d'aller au-delà de l'apparent, des lieux communs, des discours et d'analyser en profondeur de ce qu'on pourrait nommer aujourd'hui le mal-être brésilien. L'ensemble des économies 
dites du tiers-monde connaissent des évolutions économiques différentes. Certaines connaissent des taux de croissance très élevés et, tout en permettant des niveaux de vie moyens encore très éloignés de ceux existant dans les pays à économies dites développées, s'en rapprochent. Cette trajectoire n'est pas, déjà, sans poser de très nombreux problèmes en termes d'équité, voire d'éthique, de défense des droits humains, de cohésion sociale. Mais si les économies qui ne parviennent pas à « décoller » connaissent, elles, dans une certaine mesure, les mêmes problèmes, c'est dans un contexte totalement différent: au lieu de commencer à converger vers les économies développées, elles s'en éloignent et les perspectives de mobilité sociale s'éloignant, se développent non seulement la violence mais aussi les menaces de guerre civile.

Le Brésil est dans un entre-deux. Il a d'énormes potentialités mais des inégalités gigantesques et des intérêts «bien compris » suffisamment puissants pour bloquer leur remise en cause. Mieux, il les consolide en leur donnant la base financière qu'ils n'avaient pas ou peu. La violence, déjà forte dans les grandes villes, se développe et mine la cohésion sociale. Pour autant, la nation auriverde ${ }^{11}$ reste encore dans le «peloton de tête ", mais à la dernière place. Le second mandat du président Lula da Silva est décisif : soit le Brésil décroche et plonge dans les affres que connaissent déjà certains pays, soit il mute et par là consolide le processus de démocratisation entamée en 1979 avec l'abertura.

Juin 2007
Brigitte LACHARTRE

Comité de rédaction de la revue Lusotopie

Pierre SALAMA

Université Paris 13

Centre d'économie de l'Université Paris Nord (CEPN)

11 On le sait, l'or et le vert sont les deux couleurs du drapeau national. 


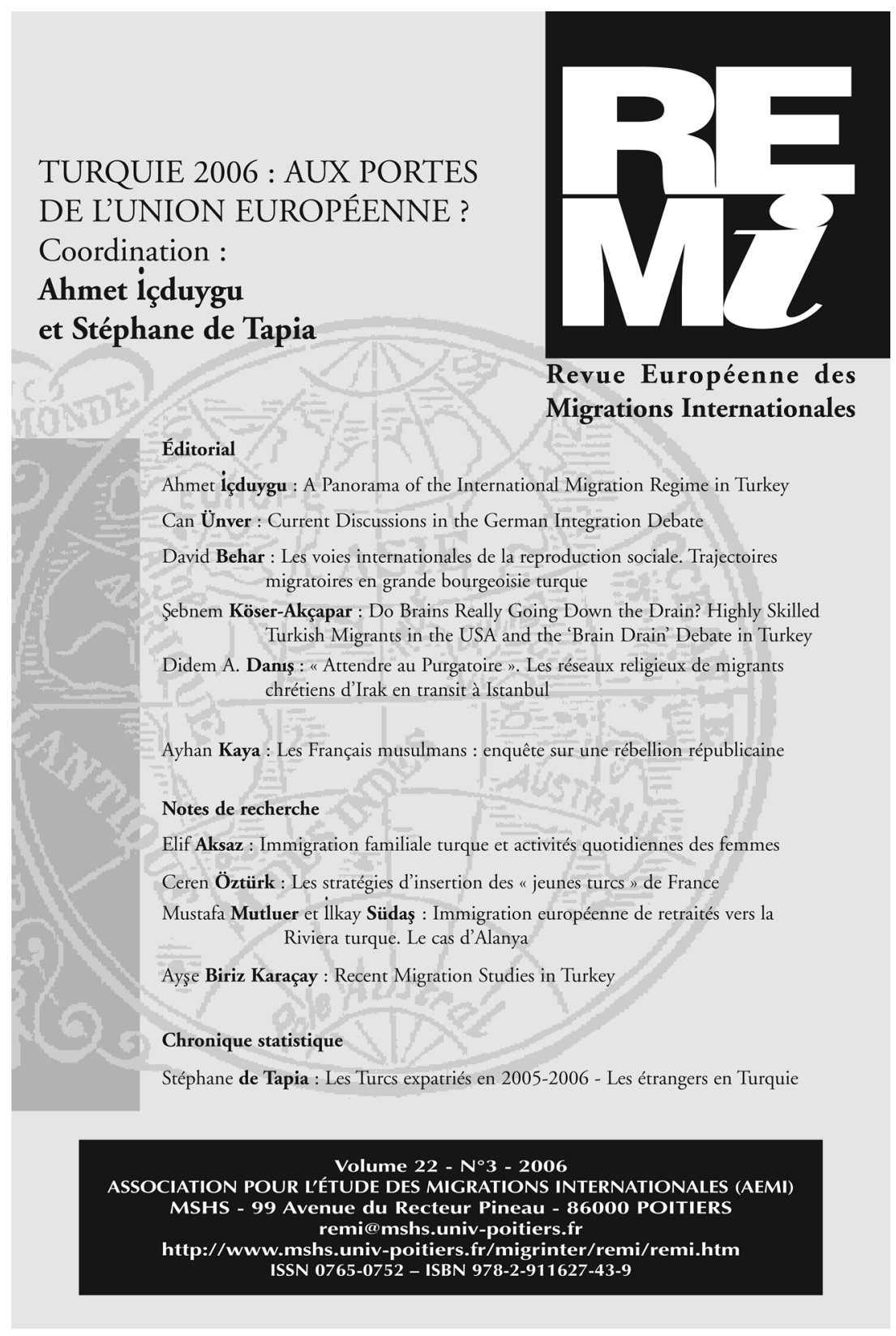

